\title{
Inadequação do conteúdo da assistência pré-natal e fatores associados em uma coorte no nordeste brasileiro
}

\author{
Inadequacy of the content of prenatal care and associated factors \\ in a cohort in the northeast of Brazil
}

\author{
Marivanda Julia Furtado Goudard ${ }^{1}$ \\ Vanda Maria Ferreira Simões ${ }^{1}$ \\ Rosângela Fernandes Lucena Batista ${ }^{1}$ \\ Rejane Christine de Souza Queiroz ${ }^{1}$ \\ Maria Tereza Seabra Soares de Brito e Alves ${ }^{1}$ \\ Liberata Campos Coimbra ${ }^{1}$ \\ Marília da Glória Martins ${ }^{2}$ \\ Marco Antônio Barbieri ${ }^{3}$ \\ Ian Favero Nathasje ${ }^{4}$
}

\begin{abstract}
The scope of this study was to analyze the content of prenatal care in São Luís, Maranhão, Brazil, and the factors associated with its inadequacy. A cross-sectional study was conducted based on data from the birth cohort of São Luis in 2010. The content of prenatal care was defined as inadequate when it did not meet the criteria of the Program for Humanization of Prenatal and Delivery Care, which establishes early initiation of prenatal care, minimum number of medical consultations, basic laboratory tests, tetanus vaccination and obstetric procedures. Poisson regression was used to observe associations of the variables with the outcome. The inadequacy rate was high (60.2\%). The variables associated with inadequacy were: class $C$ socioeconomic status $(P R=1.39$; $C I=1.26-1.55) ;$ class $D / E$ socioeconomic status $(P R=1.60 ; C I=1.43-1.79) ;$ unqualified/unemployed mother $(P R=1.24 ; C I=1.11-1.37) ; 5-8$ years of schooling $(P R=1.12 ; C I=1.06-1.19)$; $0-4$ years of schooling $(P R=1.13 ; C I=1.01$ $1.26)$; not being religious $(P R=1.10 ; C I=1.04$ 1.17); alcohol use during pregnancy $(P R=1.13$; $C I=1.06-1.20)$, and being attended by the public service $(P R=1.75 ; C I=1.54-2.00)$. The results showed inadequacy and inequality of prenatal care, revealing that women of lower socioeconomic status received lower quality care.
\end{abstract}

Key words Prenatal care, Quality of health care, Socioeconomic factors
Resumo O objetivo desta pesquisa foi analisar o conteúdo da assistência pré-natal em São Luís e os fatores associados com sua inadequação. Realizou-se estudo transversal baseado em dados da coorte de nascimento de São Luís em 2010. O conteúdo da assistência pré-natal foi classificado como inadequado quando não atendeu aos critérios do Programa de Humanização no Pré-Natal e Nascimento (PHPN) que estabelece o início precoce, o número mínimo de consultas, os exames laboratoriais básicos, a vacinação antitetânica e os procedimentos obstétricos. Regressão de Poisson foi utilizada para observar associações das variáveis com o desfecho. A taxa de inadequação foi de 60,2\%. O pré-natal inadequado foi associado à classe econômica $C(R P=1,39 ; I C=1,26-1,55)$, à $D / E(R P=1,60 ; I C=1,43-1,79)$, à ocupação da mãe não qualificada/desempregada $(R P=1,24$; $I C=1,11-1,37)$, à escolaridade de 5-8 anos ( $R P$ $=1,12 ; I C=1,06-1,19)$ e de $0-4$ anos $(R P=1,13$; $I C=1,01-1,26)$, a não ter religião $(R P=1,10 ; I C$ =1,04-1,17), ao uso de álcool durante a gestação $(R P=1,13 ; I C=1,06-1,20)$ e ao atendimento no serviço público $(R P=1,75 ; I C=1,54-2,00)$. Os resultados demonstraram inadequação e iniquidade da assistência pré-natal, evidenciando que mulheres de pior condição socioeconômica foram as que receberam assistência de menor qualidade. Palavras-chave Assistência pré-natal, Qualidade da assistência, Fatores socioeconômicos 


\section{Introdução}

A assistência pré-natal permite a identificação e o manejo de condições clínicas, sociodemográficas e de fatores comportamentais de risco nas gestações que favorece a prevenção de eventos indesejáveis à mãe e ao recém-nascido ${ }^{1}$.

Apesar de vários estudos demonstrarem a associação dessa assistência com menor grau de morbimortalidade materna e infantil, ainda persiste a preocupação, principalmente nos países em desenvolvimento, com a frequência com que mulheres e crianças morrem em decorrência da gravidez e do parto ${ }^{1-3}$.

No Brasil, em junho de 2000, foi criado, pelo Ministério da Saúde, o Programa de Humanização no Pré-Natal e Nascimento (PHPN), que preconizou os parâmetros mínimos para uma adequada assistência pré-natal, objetivando reduzir as altas taxas de morbimortalidade materna e perinatal do país, ampliar o acesso ao pré-natal, estabelecer critérios para qualificar as consultas e promover o vínculo entre a assistência ambulatorial e o parto ${ }^{4,5}$.

O PHPN buscou redefinir as bases conceituais e práticas assistenciais da atenção pré-natal no país. Estabeleceu, através da portaria 569/2000, o número mínimo de seis consultas para cada mulher, o que consistia num aumento de 50\% na média nacional já alcançada de quatro. Estabeleceu também um pacote mínimo de exames laboratoriais (hematócrito/hemoglobina, glicemia de jejum, exame de sífilis, tipo sanguíneo, exame simples de urina e oferta de anti-HIV). Recomendava uma consulta no puerpério até 42 dias após o parto, além de imunização antitetânica, atividades educativas e classificação de risco com garantia de vínculo às unidades de referências ${ }^{5,6}$.

Decorrido dois anos de implantação do programa, um estudo de avaliação observou que as mulheres cadastradas que realizaram seis ou mais consultas no pré-natal correspondiam a $22,6 \%$ no país e quando se agregava os demais critérios assistenciais (realização dos exames básicos e consulta puerperal), este percentual decrescia para $5,6 \%$, indicando que era no conjunto das atividades que residia o maior desafio para a adequação dessa assistência ${ }^{4}$.

Em 2005, o Ministério da Saúde publicou um manual técnico sobre a atenção qualificada e humanizada no pré-natal e puerpério, baseado nas recomendações do PHPN e orientou procedimentos clínicos obstétricos a serem realizados nas consultas ${ }^{7}$. Esse instrumento foi uma estratégia para alcançar as metas estabelecidas no Pacto
Nacional para Redução da Mortalidade Materna e Neonatal, lançado em 2004, que buscava uma redução de $75 \%$ nessas taxas de mortalidade para o ano de 2015, comparada com as de $1990^{8}$.

$\mathrm{O}$ acesso à assistência pré-natal de fato foi ampliado após o PHPN. Segundo a Pesquisa Nacional de Demografia e Saúde (PNDS), de 2006, a cobertura do pré-natal foi considerada praticamente universal no Brasil ${ }^{9}$. Resultado semelhante foi demonstrado por Viellas et al. em estudo de abrangência nacional no período de 2011 a 2012, quando $98,7 \%$ das gestantes realizaram consulta pré-natal e 73,1\% realizaram as 6 consultas recomendadas pelo Ministério da Saúde. Observaram também que os valores de cobertura pré-natal se mostraram elevados (superior a $90 \%$ ) em todas as regiões do país e em mulheres de diferentes características demográficas, sociais e reprodutivas ${ }^{10}$. Apesar da alta cobertura dessa assistência já alcançada, preocupa a qualidade da atenção praticada.

Buscando avaliar o conteúdo da assistência pré-natal oferecido às mulheres brasileiras surgiram estudos nacionais utilizando os critérios do PHPN como indicador de qualidade. A utilização desses critérios representou um avanço para o monitoramento do atendimento realizado, pois, além de medir o número de consultas, são introduzidos aspectos qualitativos do conteúdo dessa assistência. Essa preocupação em utilizar indicadores mais abrangentes está surgindo na literatura atual ${ }^{11-13}$. Apesar disso, ainda não há consenso sobre a quantidade de cuidados que uma mulher deve receber e qual o conteúdo que se faz estritamente necessário. Não há, portanto, uniformidade entre esses indicadores.

Estudos nacionais baseados nos parâmetros do PHPN observaram uma baixa adequação da assistência pré-natal no país. No município do Rio de Janeiro, Domingues et al. ${ }^{14}$ observaram uma taxa de $61,5 \%$ de inadequação dessa assistência. As mulheres que tiveram menor número de consultas apresentavam menor adequação de exames, vacinação antitetânica e orientações sobre o aleitamento materno.

Em Caxias do Sul, outro estudo encontrou inadequação de 64,8\% em uma amostra de gestações de baixo risco. A maior escolaridade e a menor paridade materna mostraram-se como fatores determinantes para a adequação do prénatal, nesse estudo ${ }^{3}$.

Em Recife, no ano de 2004, Carvalho e Araújo ${ }^{15}$ observaram alta inadequação da assistência pré-natal $(82,2 \%)$ pelo PHPN em uma amostra de gestantes de alto risco, sendo o perfil socioeco- 
nômico dessa amostra formado, em maior proporção, por jovens, vivendo com o parceiro, com baixa renda e menor escolaridade. A baixa renda também foi um fator associado com inadequação do pré-natal num estudo em Campinas, cuja amostra era representada por uma proporção maior de gestantes adolescentes, negras ou pardas, solteiras e com menor escolaridade ${ }^{16}$.

Em São Luís, capital do Maranhão, foi avaliada a adequação da assistência pré-natal por mulheres participantes de uma coorte em 1997/1998, utilizando, porém, outro indicador, baseado no início e no número de consultas realizadas, ou seja, investigando aspectos quantitativos da assistência. A taxa de inadequação observada foi de $34,6 \%$, sendo associada a mulheres atendidas em hospitais públicos, sem companheiro, com alta paridade e com menores níveis de escolaridade e renda familiar ${ }^{17}$.

O Maranhão é um dos estados do Nordeste brasileiro, registrando o segundo pior índice de desenvolvimento humano do país ${ }^{18}$. Como vem ocorrendo no país como um todo, o Maranhão também tem elevado suas taxas de cobertura de pré-natal, fato observado no estudo de Costa et al. ${ }^{19}$, cuja taxa foi de $85,6 \%$ das mulheres pesquisadas em 30 municípios do Estado, no período de 2007 a 2008.

A alta cobertura da assistência pré-natal observada no Maranhão está associada com a incorporação, na prática clínica, dos critérios de qualidade recomendados pelo PHPN em sua capital? A presente pesquisa, estudo populacional em São Luís, objetiva responder a este questionamento, assim como identificar quais fatores estariam associados com uma possível inadequação do conteúdo dessa assistência.

\section{Métodos}

Trata-se de um estudo transversal aninhado a uma coorte de nascimento intitulada "Fatores etiológicos do nascimento pré-termo e consequências dos fatores perinatais na saúde da criança: coortes de nascimentos em duas cidades brasileiras - BRISA" que foi desenvolvido pela Universidade de São Paulo (USP) e pela Universidade Federal do Maranhão (UFMA), em dois municípios brasileiros - Ribeirão Preto, SP e São Luís, MA.

Neste estudo foram utilizados os dados de São Luís, capital do Estado do Maranhão, cuja população em 2010 era de 1.014 .837 habitantes ${ }^{20}$. Localiza-se em uma das regiões mais pobres do país, seu último índice de desenvolvimento hu- mano (IDH) publicado foi de 0,768, levando-a a posição de $249^{\circ}$ entre os municípios do Brasil ${ }^{20}$.

A coorte de base populacional de São Luís foi formada ao longo dos meses de janeiro a dezembro do ano de 2010, e incluiu os nascimentos em instituições de saúde públicas e privadas que realizavam mais de 100 partos por ano. No período do estudo, 98\% dos nascimentos ocorreram em hospitais. As instituições que realizavam menos de 100 partos por ano correspondeu a 3,3\% dos nascimentos e não foram incluídas no estudo. Sendo assim, a população alvo consistiu de $94,7 \%$ de todos os nascimentos.

A amostra do estudo foi estratificada por unidade de saúde com partilha proporcional ao número de partos e em cada unidade ela foi sistemática. Todos os nascimentos foram listados por ordem de ocorrência. Um número aleatório de 1 a 3 foi gerado para determinar o ponto de início do estudo em cada estabelecimento. O intervalo amostral determinado foi 3 (três). Assim, um em cada três nascimentos foi escolhido aleatoriamente para a entrevista. Do total de 7.133 nascimentos selecionados, 5.475 eram de mulheres residentes no município há pelo menos três meses e, portanto, elegíveis. Após 4,6\% de perdas por recusas da mãe em participar ou devido a alta precoce, resultou em uma amostra de 5.236 nascimentos.

Para alcançar o objetivo do estudo também foram excluídos os óbitos e as gestações gemelares, totalizando 5.067 nascimentos de parto único. No entanto, o indicador do PHPN não foi possível ser aplicado nas puérperas que não realizaram consultas de pré-natal e que não recordaram de informações necessárias como dia e mês da última menstruação, solicitação de exames laboratoriais, realização de vacina antitetânica e/ ou de procedimentos clínicos obstétricos, sendo excluídas da análise 1.118 mulheres. Portanto, para este estudo utilizou-se uma amostra total de 3.949 puérperas.

Para o estudo da adequação da assistência pré-natal, o tamanho mínimo da amostra foi fixado em 2.155 nascimentos, considerando uma estimativa de prevalências por volta de $50 \%$, uma precisão de $2 \%$ e nível de confiança de $95 \%$. Com um tamanho de amostra de 3.210 é também possível comparar duas proporções em torno de $50 \%$, considerando probabilidade de erro tipo I de $5 \%$, o poder do estudo de $80 \%$, e fixando em $5 \%$ a diferença mínima a ser detectada como significante.

Um questionário padronizado foi aplicado às mulheres, preferencialmente nas primeiras 24 horas após o parto. Informações sobre a assistência 
pré-natal foi obtida por meio desses questionários e dos prontuários maternos, quando disponível.

O desfecho do presente estudo foi a adequação do conteúdo da assistência pré-natal baseado nos critérios do $\mathrm{PHPN}^{5}$ e do manual do Pré-Natal e Puerpério do Ministério da Saúde 7 . Foi considerado adequado quando a gestante iniciou o pré-natal até o quarto mês de gestação; realizou, no mínimo, seis consultas para uma gestação a termo ( $\geq 37$ semanas) ou um menor número de acordo com a idade gestacional ao parto (três consultas até 29 semanas; quatro consultas de 30 a 33 semanas e cinco consultas de 34 a 36 semanas); confirmou solicitação dos exames básicos de rotina (exame de sangue para hematócrito/hemoglobina e glicemia, exame do tipo sanguíneo, exame de sífilis, exame de urina, anti-HIV); realizou vacinação antitetânica anterior ou vacinação na gestação atual; confirmou ter sido aferida sua pressão arterial, seu peso e a medida da altura do fundo uterino; cálculo da idade gestacional por meio da data da última menstruação e exame das mamas. Foi considerado inadequado às demais situações.

Como o estudo foi realizado nos primeiros dias após o parto, o componente consulta puerperal não foi incluído. Foi considerado que o dado "realização de exame de sangue", contemplou minimamente os exames de hemograma (hematócrito, hemoglobina) e glicemia que são habitualmente solicitados na primeira consulta. Foi imputado $15^{\circ}$ dia do mês, para efeito do cálculo da idade gestacional, quando a puérpera não lembrou o dia exato da data da última menstruação.

As variáveis independentes foram divididas em três níveis, a partir de um modelo teórico hierarquizado, segundo preconizado por alguns autores ${ }^{21,22}$. Neste modelo assumiu-se que variáveis socioeconômicas e culturais influenciam nas características familiares (incluindo na idade em que essa mulher se torna mãe) que, por sua vez, influenciam nas reprodutivas, assim como nas morbidades e nos comportamentos de risco dessa mulher, além do tipo de serviço onde ela recebeu sua assistência pré-natal, que, por sua vez, influenciam no desfecho (Figura 1).

No nível 1 foram incluídas como variáveis distais os fatores socioeconômicos: escolaridade (0-4, 5-8 e 9 ou mais anos de estudo), ocupação da mãe (trabalho não manual de nível médio ou superior, manual qualificado ou semiespecializado, manual não qualificado ou desempregada) além da classificação econômica da família segundo o Critério de Classificação Econômica Brasil, desenvolvido pela Associação Brasileira de
Empresas de Pesquisa ${ }^{23}$ ( categorizado em Classe A/B; C e D/E ); variável demográfica: cor da pele autorreferida (branca, preta, parda ou outra) e característica cultural: ter religião (sim ou não).

No nível 2, intermediário, foram incluídas as características familiares: idade da mãe $(<20,20$ 34 e 35 a mais anos) e situação conjugal (casada, união consensual, sem companheiro).

No nível 3, como variáveis proximais foram incluídas as características reprodutivas de paridade (primeiro filho, 2 a 4 filhos e 5 ou mais filhos), filhos anteriores prematuros (sim ou não) e natimortos anteriores (sim ou não); as morbidades maternas hipertensão gestacional (sim ou não), diabetes gestacional (sim ou não); as características comportamentais de tabagismo na gestação (sim ou não), uso de álcool na gestação (sim ou não); características do serviço de saúde onde realizou o pré-natal (público ou privado) e se houve assistência pela Estratégia Saúde da Família-ESF (sim ou não).

A digitação dos dados foi realizada em duplicata no programa Microsoft Office Access 2007. A análise estatística foi feita no programa Stata 12.0 (Stata Corporation, College Station, Texas, EUA).

A análise estatística foi realizada por meio de regressão múltipla de Poisson com ajuste robusto da variância, a partir do modelo teórico hierarquizado pré-concebido. Iniciou-se a análise com o nível mais distal, no qual variáveis com $\mathrm{p}<0,20$ foram introduzidas nos níveis seguintes como fatores de ajuste. Procedeu-se da mesma forma com o nível intermediário, permanecendo as variáveis com $\mathrm{p}<0,20$ como fatores de ajuste para o nível proximal. Finalmente, mantidos os fatores de ajuste dos níveis 1 e 2 , foram introduzidas as variáveis do nível 3, permanecendo no modelo final as com $\mathrm{p}<0,20$. Foram consideradas associadas ao desfecho as variáveis que apresentaram p $<0,05$, com IC de 95\%, dentro de cada nível.

O estudo atendeu aos critérios da Resolução 466/2012 do Conselho Nacional de Saúde, tendo sido aprovado pelo Comitê de Ética em Pesquisa do Hospital Universitário da Universidade Federal do Maranhão.

\section{Resultados}

Do total de 5.067 nascimentos de partos únicos da coorte de nascimento de São Luís-MA em 2010, foram excluídas da análise 1.118 puérperas por não haver informações suficientes para a utilização do indicador de conteúdo. A população incluída no estudo totalizou 3.949 mulheres. 




Figura 1. Modelo hierarquizado para a análise da adequação do conteúdo da assistência pré-natal.

Não houve diferença significativa entre a população incluída e a excluída do estudo em relação às variáveis: idade, cor da pele, ocupação da mãe, situação conjugal, hipertensão e diabetes gestacional, natimorto anterior, local de atendimento do pré-natal e atendimento na ESF. Houve diferença em relação à escolaridade, classe econômica, paridade, prematuridade anterior, ter religião, tabagismo e álcool durante a gestação. A maior proporção de anos de estudo, de classe A/B e C, primíparas, religiosas e não usuárias de drogas lícitas pertenceram ao grupo das mulheres incluídas no estudo (Tabela1).

A população estudada foi constituída, em maior frequência, por adultas jovens de 20 a 34 anos $(74,0 \%)$, pardas $(67,9 \%)$, com mais de 9 anos de estudo (75,3\%), vivendo em união consensual $(59,3 \%)$, de classe econômica C (55,2\%), com ocupação da mulher possuindo trabalho manual não qualificado/desempregada $(79,8 \%)$, com paridade de 2 a 4 gestações $(46,5 \%)$, tendo religião $(81,6 \%)$, sem filho prematuro anterior $(86,6 \%)$, sem natimorto anterior $(97,4 \%)$, não fumantes $(96,9 \%)$, sem uso de álcool $(86,2 \%)$, sem hipertensão gestacional $(83,6 \%)$, sem diabetes gestacional $(97,9 \%)$, com atendimento do pré-natal no sistema público de saúde (81,6\%) e $89,1 \%$ sem assistência da Estratégia Saúde da Família (Tabela 1).

Utilizando o indicador de conteúdo de adequação observou-se que $1.572(39,8 \%)$ gestantes realizaram assistência pré-natal adequada e $2.377(60,2 \%)$ de forma inadequada (dado não demonstrado em tabela).

O conteúdo da assistência pré-natal que refletiu maior prejuízo nas taxas de utilização foi relacionado aos critérios de não adequação do número de consultas por idade gestacional 
Tabela 1. Características socioeconômicas, demográficas, reprodutivas e comportamentais das mulheres incluídas e excluídas da análise na coorte populacional BRISA, São Luís, 2010.

\begin{tabular}{|c|c|c|c|c|c|}
\hline Características & $\begin{array}{l}\text { Incluídas } \\
\text { n (3.949) }\end{array}$ & $(\%)$ & $\begin{array}{l}\text { Excluídas } \\
\text { n (1.118) }\end{array}$ & $(\%)$ & $\mathrm{p}$ valor \\
\hline Idade (anos) & & & & & 0,403 \\
\hline$<20$ & 721 & 18,3 & 224 & 20,0 & \\
\hline $20-34$ & 2.923 & 74,0 & 810 & 72,4 & \\
\hline$\geq 35$ & 305 & 7,7 & 84 & 7,5 & \\
\hline Cor da pele & & & & & 0,514 \\
\hline Branca & 711 & 18,0 & 221 & 19,8 & \\
\hline Preta & 499 & 12,6 & 145 & 12,9 & \\
\hline Parda & 2.682 & 67,9 & 734 & 65,9 & \\
\hline Outra & 57 & 1,4 & 15 & 1,3 & \\
\hline Escolaridade (anos de estudo) & & & & & $<0,001$ \\
\hline $0-4$ & 136 & 3,4 & 94 & 8,5 & \\
\hline $5-8$ & 838 & 21,3 & 291 & 26,2 & \\
\hline$\geq 9$ & 2.968 & 75,3 & 726 & 65,3 & \\
\hline Ocupação da mãe & & & & & 0,169 \\
\hline Trabalho não manual & 450 & 11,9 & 110 & 10,2 & \\
\hline Manual semiespecializado & 306 & 8,1 & 79 & 7,3 & \\
\hline Manual não qualificado/desempregada & 2.999 & 79,8 & 886 & 82,4 & \\
\hline Situação conjugal & & & & & 0,197 \\
\hline Casada & 875 & 22,2 & 230 & 20,6 & \\
\hline União consensual & 2.341 & 59,3 & 656 & 58,7 & \\
\hline Sem companheiro & 733 & 18,5 & 232 & 20,7 & \\
\hline Ter religião & & & & & 0,049 \\
\hline Sim & 3.222 & 81,6 & 883 & 79 & \\
\hline Não & 722 & 18,4 & 235 & 21 & \\
\hline Paridade ( $n^{\circ}$ de filhos) & & & & & $<0,001$ \\
\hline 1 & 2.002 & 50,7 & 410 & 36,7 & \\
\hline $2-4$ & 1.835 & 46,5 & 645 & 57,7 & \\
\hline$\geq 5$ & 112 & 2,8 & 63 & 5,6 & \\
\hline Filho prematuro anterior & & & & & $<0,001$ \\
\hline Não & 3.408 & 86,6 & 913 & 81,9 & \\
\hline Sim & 529 & 13,4 & 201 & 18,1 & \\
\hline Natimorto anterior & & & & & 0,104 \\
\hline Não & 3.847 & 97,4 & 1.079 & 96,5 & \\
\hline Sim & 102 & 2,6 & 39 & 3,5 & \\
\hline Tabagismo durante a gestação & & & & & $<0,001$ \\
\hline Não & 3.825 & 96,9 & 1.038 & 92,8 & \\
\hline Sim & 124 & 3,1 & 80 & 7,2 & \\
\hline Álcool durante a gestação & & & & & 0,007 \\
\hline Não & 3.404 & 86,2 & 928 & 83,0 & \\
\hline $\operatorname{Sim}$ & 545 & 13,8 & 190 & 17,0 & \\
\hline Hipertensão gestacional & & & & & 0,741 \\
\hline Não & 3.299 & 83,6 & 938 & 83,9 & \\
\hline Sim & 649 & 16,4 & 179 & 16,1 & \\
\hline Diabetes gestacional & & & & & 0,524 \\
\hline Não & 3.868 & 97,9 & 1.087 & 97,7 & \\
\hline Sim & 80 & 2,0 & 26 & 2,3 & \\
\hline Classe Econômica & & & & & $<0,001$ \\
\hline A & 104 & 2,8 & 39 & 3,0 & \\
\hline B & 633 & 16,9 & 137 & 13,4 & \\
\hline $\mathrm{C}$ & 2.066 & 55,2 & 498 & 48,6 & \\
\hline $\mathrm{D}$ & 808 & 21,6 & 290 & 28,3 & \\
\hline $\mathrm{E}$ & 129 & 3,4 & 61 & 5,9 & \\
\hline Local de atendimento do pré-natal & & & & & 0,735 \\
\hline Privado & 725 & 18,4 & 183 & 17,9 & \\
\hline Público & 3.215 & 81,6 & 837 & 82,1 & \\
\hline Atendimento pela ESFa & & & & & 0,590 \\
\hline Não & 3.516 & 89,1 & 988 & 88,5 & \\
\hline Sim & 430 & 10,9 & 128 & 11,5 & \\
\hline
\end{tabular}

p valor pelo teste Qui-quadrado. ${ }^{a}$ Estratégia Saúde da Família. Total de algumas variáveis diferentes do n devido às respostas ignoradas. 
Tabela 2. Indicador de Conteúdo da Qualidade da assistência pré-natal na coorte populacional BRISA, São Luís, 2010.

\begin{tabular}{lrr}
\hline \multicolumn{1}{c}{ Critério de qualidade } & \multicolumn{1}{c}{$\begin{array}{c}\text { Sim } \\
\mathbf{n}(\%)\end{array}$} & \multicolumn{1}{c}{$\begin{array}{c}\text { Não } \\
\text { n (\%) }\end{array}$} \\
\hline - Início do pré-natal até o 4o mês & $4.153(82,1)$ & $914(18,0)$ \\
- Adequação de n ${ }^{\circ}$ consultas por idade gestacional & $3.174(66,0)$ & $1.633(34,0)$ \\
- Solicitação de exame de sangue & $4.928(99,2)$ & $40(0,8)$ \\
- Solicitação de anti-HIV & $4.792(96,5)$ & $172(3,5)$ \\
- Solicitação de exame de urina & $4.890(98,4)$ & $78(1,6)$ \\
- Solicitação do tipo sanguíneo & $4.705(95,0)$ & $247(5,0)$ \\
- Solicitação do exame para sífilis (VDRL) & $4.645(94,2)$ & $287(5,8)$ \\
- Vacinação antitetânica & $4.631(91,6)$ & $424(8,4)$ \\
- Verificação do peso & $4.923(99,1)$ & $43(0,9)$ \\
- Exame da altura do fundo uterino & $4.850(97,66)$ & $116(2,3)$ \\
- Verificação da pressão arterial & $4.917(99,0)$ & $47(0,9)$ \\
- Exame das mamas & $3.118(62,8)$ & $1.844(37,2)$ \\
- Verificação da data da última menstruação para cálculo da idade gestacional & $4.932(99,3)$ & $35(0,7)$ \\
& &
\end{tabular}

O n de alguns dos critérios difere do $\mathrm{n}$ inicial (5.067) devido às respostas ignoradas.

$(34,0 \%)$, não realização do exame das mamas $(37,2 \%)$ e não iniciar o pré-natal até o quarto mês de gestação (18,0\%) (Tabela 2).

Após a análise hierarquizada e ajustada, as variáveis que permaneceram significantes ( $\mathrm{p}<$ $0,05)$ com a inadequação do conteúdo da assistência pré-natal foram: no nível distal a classe econômica $\mathrm{C}(\mathrm{RP}=1,39$; $\mathrm{IC}=1,26-1,55)$, $\mathrm{D} / \mathrm{E}$ $(\mathrm{RP}=1,60 ; \mathrm{IC}=1,43-1,79)$, ocupação da mãe não qualificada/desempregada $(\mathrm{RP}=1,24$; $\mathrm{IC}$ $=1,11-1,37)$, a escolaridade de 5-8 anos ( $\mathrm{RP}=$ 1,12 ; IC = 1,06-1,19), de 0-4 anos ( $\mathrm{RP}=1,13$; IC $=1,01-1,26)$ e não ter religião $(\mathrm{RP}=1,10$; $\mathrm{IC}=$ 1,04-1,17); no nível intermediário a idade de 35 anos ou mais $(\mathrm{RP}=0,86$; $\mathrm{IC}=0,76-0,98)$; no nível proximal a primiparidade $(\mathrm{RP}=0,82$; $\mathrm{IC}=$ 0,77-0,87), uso de álcool durante a gestação (RP $=1,13 ; \mathrm{IC}=1,06-1,20)$ e atendimento no serviço público $(\mathrm{RP}=1,75 ; \mathrm{IC}=1,54-2,00)$ (Tabela 3$)$.

\section{Discussão}

Este estudo observou uma alta taxa de inadequação $(60,2 \%)$ do conteúdo da assistência pré-natal realizado pelas gestantes de São Luís, mostrando associação com baixa classe econômica, trabalho não qualificado/desempregada, baixa escolaridade, usuárias de álcool na gestação, sem religião e que fizeram atendimento pré-natal em serviço público de saúde. A primiparidade e a idade de 35 anos ou mais demonstraram menores taxas de inadequação dessa assistência. Os itens do conteúdo da assistência pré-natal com menores taxas de utilização foram número de consultas por idade gestacional, exame das mamas e início do pré-natal até o quarto mês de gestação.

Estudos que objetivaram observar a assistência pré-natal para além do número de consultas realizadas, utilizando indicadores para avaliar o conteúdo dessa assistência têm encontrado um percentual de inadequação mais elevado em relação aos indicadores tradicionais. Em Bruxelas, estudo de Beekman et al. ${ }^{11}$, baseado em um indicador que aferiu o início do pré-natal e o número total de consultas por idade gestacional, observou que apenas 2,4\% das gestações tiveram um prénatal inadequado, porém quando comparado com um indicador de conteúdo que acrescentava a aferição de pressão arterial, exames de sangue e ultrassonografia, essa inadequação subiu para 10,2\%. Em outro estudo, na Turquia, a taxa de inadequação observada baseada na quantidade de consultas e início do pré-natal foi de 35,1\% 
Tabela 3. Análise hierarquizada ajustada por regressão de Poisson dos fatores associados ao conteúdo inadequado da assistência pré-natal na coorte populacional BRISA. São Luís, 2010.

\begin{tabular}{|c|c|c|c|}
\hline Variável & $\mathbf{R P}$ & IC $95 \%$ & p valor \\
\hline \multicolumn{4}{|l|}{ Nível 1 - Distal } \\
\hline Classe Econômica & & & $<0,001$ \\
\hline $\mathrm{A} / \mathrm{B}$ & 1 & & \\
\hline $\mathrm{C}$ & 1,39 & $1,26-1,55$ & \\
\hline $\mathrm{D} / \mathrm{E}$ & 1,60 & $1,43-1,79$ & \\
\hline Ocupação da mãe & & & $<0,001$ \\
\hline Trabalho não manual & 1 & & \\
\hline $\begin{array}{l}\text { Manual } \\
\text { semiespecializado }\end{array}$ & 1,02 & $0,88-1,19$ & \\
\hline $\begin{array}{l}\text { Não qualificado/ } \\
\text { desempregada }\end{array}$ & 1,24 & $1,11-1,37$ & \\
\hline Cor da mãe & & & 0,152 \\
\hline Branca & 1 & & \\
\hline Preta & 1,06 & $0,96-1,17$ & \\
\hline Parda & 1,07 & $0,99-1,16$ & \\
\hline Escolaridade (em anos) & & & $<0,001$ \\
\hline 9 ou mais & 1 & & \\
\hline 5 a 8 & 1,12 & $1,06-1,19$ & \\
\hline 0 a 4 & 1,13 & $1,01-1,26$ & \\
\hline Ter religião & & & 0,001 \\
\hline Sim & 1 & & \\
\hline Não & 1,10 & $1,04-1,17$ & \\
\hline \multicolumn{4}{|l|}{ Nível 2 - Intermediário* } \\
\hline Idade (em anos) & & & 0,049 \\
\hline $20-34$ & 1 & & \\
\hline$<20$ & 1,02 & $0,96-1,08$ & \\
\hline 35 ou mais & 0,86 & $0,76-0,98$ & \\
\hline Situação conjugal & & & 0,161 \\
\hline Casada & 1 & & \\
\hline União consensual & 1,07 & $0,99-1,16$ & \\
\hline Sem companheiro & 1,08 & $0,99-1,18$ & \\
\hline \multicolumn{4}{|l|}{ Nível 3 - Proximal ${ }^{* *}$} \\
\hline Paridade ( ${ }^{\circ}$ filhos) & & & $<0,001$ \\
\hline $2-4$ & 1 & & \\
\hline 1 & 0,82 & $0,77-0,87$ & \\
\hline 5 ou mais & 1,10 & $0,99-1,23$ & \\
\hline $\begin{array}{l}\text { Uso de álcool na } \\
\text { gestação }\end{array}$ & & & $<0,001$ \\
\hline Não & 1 & & \\
\hline Sim & 1,13 & $1,06-1,20$ & \\
\hline $\begin{array}{l}\text { Local de atendimento } \\
\text { do pré-natal }\end{array}$ & & & $<0,001$ \\
\hline Privado & 1 & & \\
\hline Público & 1,75 & $1,54-2,00$ & \\
\hline
\end{tabular}

RP = Razão de Prevalência. IC (Intervalo de Confiança). * Modelo ajustado também para as variáveis do nível proximal com $\mathrm{p}<0,20$. * Modelo ajustado também para as variáveis dos níveis proximal e intermediário com $\mathrm{p}<0,20$.

e, após utilização de um indicador de conteúdo (que incluía verificar o peso e pressão arterial da gestante, ausculta de batimentos cardíacos fetais, exames laboratoriais, imunização antitetânica e conselhos sobre estilo de vida saudável), a taxa aumentou para $74,1 \%{ }^{12}$.

As taxas de inadequação do conteúdo da assistência pré-natal no Brasil são bem elevadas, baseado nos critérios do PHPN. Em um estudo de base populacional no sul do país, apesar da alta cobertura $(96 \%)$ encontrada, observou-se inadequação da assistência pré-natal em 73,2\% das gestantes entrevistadas ${ }^{2}$. Talvez esse município tenha apresentado uma taxa de inadequação maior que o presente estudo devido ao indicador utilizado ter investigado também a realização do exame colpocitológico de colo uterino e suplementação de ferro. Esse fato demonstra que quanto mais se avalia o conteúdo de cuidado prénatal que é oferecido à gestante, mais se evidencia a inadequação dessa assistência, como observado em outros estudos nacionais baseados nos parâmetros do PHPN ${ }^{3,6,14-16}$.

A última revisão sistemática sobre o desempenho do PHPN no Brasil observou o baixo cumprimento do conjunto das metas propostas em todas as regiões estudadas. Além disso, grande parte dos estudos disponíveis demonstraram realidades locais, sem possibilidade de generalização dos dados ${ }^{6}$. O presente estudo de base populacional demonstra essa tendência ao não cumprimento dos critérios propostos por esse programa em São Luís, observada pela alta taxa de inadequação encontrada, apesar de já haver completado uma década de sua implantação até a coorte de 2010.

Após análise hierarquizada ajustada observou-se, no presente estudo, as seguintes variáveis associadas com a inadequação do conteúdo da assistência pré-natal: classe econômica $\mathrm{C}$ ou D/E, ocupação da mãe não qualificada/desempregada, baixa escolaridade, álcool durante a gestação, não ter religião e utilizar o serviço público de saúde para atendimento do pré-natal.

Condições socioeconômicas desfavoráveis têm sido associadas com inadequação de assistência pré-natal em vários estudos, mesmo utilizando outros indicadores de qualidade ${ }^{1,13,14,16,17,24-27}$. As mulheres de baixas condições econômicas são as que utilizam em maior proporção o serviço público de saúde para a sua assistência pré-natal ${ }^{10,16,17,27}$. Por outro lado, os estudos vêm demonstrando que o setor privado de saúde tem oferecido uma assistência de melhor qualidade quando comparado ao público. Na Turquia, Ciceklioglu et al. ${ }^{12}$, observaram que o preditor mais importante de adequação do conteúdo dessa assistência era o uso contínuo do setor privado. No Brasil, Victora et al. ${ }^{27}$, observaram, através de um 
escore de qualidade do PHPN, maior adequação no setor privado e entre as mulheres mais ricas. O presente estudo observou uma razão de prevalência de 1,75 de inadequação para mulheres que realizaram seu pré-natal no Sistema Único de Saúde (SUS). Outros estudos nacionais também têm demonstrado que o uso do serviço público de saúde está associado à menor qualidade da assistência pré-natal ${ }^{2,15,17,28}$.

A baixa escolaridade materna é outro fator comumente associado com menor qualidade de assistência pré-natal. O menor nível educacional está geralmente relacionado com gestantes de menor condição econômica e influencia de forma negativa na utilização adequada da assistência pré-natal, fato amplamente documentado na literatura ${ }^{2,3,17,25-28}$.

Os dados apontam para uma manutenção da iniquidade da assistência à saúde reprodutiva da mulher brasileira, na qual as classes econômicas menos favorecidas apresentam maior prejuízo na qualidade dessa assistência e, apesar do aumento da cobertura, o pré-natal garantido pelo SUS, não tem sido suficiente em minimizar essa inversão dos cuidados.

O presente estudo demonstrou que fatores comportamentais e culturais podem interferir com o tipo de cuidado realizado no período gestacional. Gestantes usuárias de álcool e sem crença religiosa mostraram associação com a inadequação da qualidade do pré-natal.

O uso de álcool durante a gestação está associado a uma série de comportamentos de risco, tais como a violência contra a mulher, principalmente por parceiro íntimo, quer seja psicológica, física ou sexual ${ }^{29}$. Por outro lado, Borleider et al. ${ }^{25}$, descreveram que não ter uma rede social de apoio constituiu uma barreira para o acesso a assistência pré-natal. Conclui-se que a existência de um comportamento de risco grave agregado à falta de apoio, quer seja pelo parceiro íntimo, por familiares ou até mesmo por um grupo religioso deixam essas gestantes mais vulneráveis, afetando de forma negativa o uso adequado do seu pré-natal.

Como fatores associados à proteção da qualidade da assistência pré-natal, o presente estudo observou este fato em mulheres primíparas e com maior idade. A primiparidade tem sido associada com maior utilização da assistência prénatal em vários estudos ${ }^{3,17,26-28}$. Ciceklioglu et al. ${ }^{12}$ argumentaram que mulheres com menor paridade são mais cautelosas com suas gestações e Nketiah-Amponsah et al. ${ }^{30}$ teorizaram que as mulheres mais velhas são mais propensas a desenvolver complicações na gestação e por isso, intensificam suas consultas no pré-natal.

Buscar a equidade e a qualidade da assistência pré-natal foi o princípio norteador do PHPN, utilizando para esse fim a rede básica do SUS sob a responsabilidade, principalmente, de gestores municipais, na viabilização desse processo. No entanto, o que se tem observado nos últimos anos, por meio dos estudos que utilizaram os critérios de avaliação do PHPN, é que a garantia desse princípio ainda não foi alcançada em sua totalidade. Além do comprometimento da qualidade oferecida, ainda prevalece iniquidade dessa assistência. As mulheres de classe social mais baixa, com trabalho não qualificado/desempregada, de baixa escolaridade, as que usam álcool na gestação, sem religião, as que utilizam o serviço público de saúde, enfim, as mais fragilizadas do sistema socioeconômico, continuam sendo as que recebem assistência pré-natal de pior qualidade. A assistência pré-natal conseguiu a "universalização" junto às gestantes brasileiras, no entanto o mesmo não pode ser afirmado com relação ao conteúdo dessa assistência.

Um ponto forte do presente estudo foi ter utilizado uma coorte de nascimento, representativa da população de São Luís.

Outra vantagem foi utilizar indicador que objetiva enxergar para além da quantidade de consultas realizadas, resultando em uma imagem mais detalhada do uso da assistência pré-natal. Utilizar os critérios do PHPN representou avanço na avaliação do conteúdo dessa assistência ofertada, embora não tenham sido contemplados todos os procedimentos efetivos que deveriam ser realizados nessa assistência, conforme recomendados nos manuais técnicos do Ministério da Saúde ${ }^{7}$, posto que o instrumento da coorte de nascimento não abrangia todas essas informações.

Como limitações, houve uma elevada percentagem de puérperas $(22,1 \%)$ que não recordaram de dados importantes para que o indicador utilizado pudesse ser aplicado. Além disso, essa perda foi, em maior proporção, de mulheres de menor classe social, menor escolaridade, multíparas, fumantes e usuárias de álcool, o que pode ter subestimado a taxa de inadequação da assistência pré-natal nessa população.

Deve-se considerar a possibilidade da ocorrência do viés recordatório, que pode ocorrer em dados obtidos por meio de entrevistas e que poderia ter sido minimizado pela pesquisa no cartão da gestante e/ou no prontuário médico. No entanto, Zanchi et al. ${ }^{31}$ observaram que o sub 
-registro dos dados, comumente encontrado em prontuários e cartão de gestante, influenciam de forma negativa estudos de avaliação da qualidade do pré-natal.

Os resultados deste estudo demonstram que, de modo geral, os critérios preconizados pelo PHPN não foram incorporados à prática clínica em São Luís e que permanecem iniquidades na assistência à saúde prestada a essa população. Estudos que utilizem critérios que avaliem o conteúdo da assistência pré-natal devem ser estimulados, a fim de promover um monitoramento mais fidedigno das políticas de saúde materno-infantil que vigoram no país.

\section{Colaboradores}

MJF Goudard e VMF Simões trabalharam na concepção teórica, análise dos dados, discussão, revisão e redação final do texto. RFL Batista trabalhou na metodologia, análise estatística e revisão do texto. MTSSB Alves e IF Nathasje trabalharam na concepção teórica e estatística do instrumento utilizado como indicador do conteúdo da assistência pré-natal. MA Barbieri trabalhou na concepção do modelo teórico e na revisão final do texto. RCS Queiroz, LC Coimbra e MG Martins trabalharam na revisão e redação final do texto.

\section{Agradecimentos}

À Fundação de Amparo à Pesquisa do Estado do Maranhão (FAPEMA); Conselho Nacional de Desenvolvimento Científico e Tecnológico (CNPq); Programa de Apoio a Núcleos de Excelência (PRONEX); Fundação de Amparo à Pesquisa do Estado de São Paulo (FAPESP) e ao Hospital Universitário da Universidade Federal do Maranhão (HUUFMA). 


\section{Referências}

1. Okoroh EM, Coonrod DV, Chapple K, Drachman D. Are neonatal morbidities associated with no prenatal care different from those associated with inadequate prenatal care? Open J Obstet Gynecol 2012; 2(2):89-97.

2. Gonçalves CV, Cesar JA, Mendonza RA. Qualidade e equidade na assistência à gestante: um estudo de base populacional. Cad Saude Publica 2009; 25(11):25072516.

3. Trevisan MR, Lorenzi DS, Araújo NM, Ésber K. Perfil da Assistência Pré-Natal entre Usuárias do Sistema Único de Saúde em Caxias do Sul. Rev Bras Ginecol Obstet 2002; 24(5):293-299.

4. Serruya SJ, Lago TG, Cecatti JG. Avaliação preliminar do Programa de Humanização no Pré-Natal e Nascimento no Brasil. Rev Bras Ginecol Obstet 2004; 26(7):517-525.

5. Brasil. Ministério da Saúde (MS). Portaria no 569/GM, de 1 de junho de 2000. Institui o Programa de Humanização no Pré-Natal e Nascimento, no âmbito do Sistema Único de Saúde. Diário Oficial da União 2012; 8 jun.

6. Andreucci CB, Cecatti JG. Desempenho de indicadores de processo do Programa de Humanização do PréNatal e Nascimento no Brasil: uma revisão sistemática. Cad Saude Publica 2011; 27(6):1053-1064.

7. Brasil. Ministério da Saúde (MS). Secretaria de Atenção à Saúde. Departamento de Ações Programáticas Estratégicas. Área Técnica de Saúde da Mulher. Pré-Natal e Puerpério: atenção qualificada e humanizada. Brasília: MS; 2005.

8. Brasil. Ministério da Saúde (MS). Portaria no 427/GM, de 22 de março de 2004. Institui a Comissão Nacional de Monitoramento e Avaliação da Implementação do Pacto Nacional pela Redução da Mortalidade Materna e Neonatal e dá outras providências. Diário Oficial da União 2004; 23 mar.

9. Brasil. Ministério da Saúde (MS). Centro Brasileiro de Análise e Planejamento. Pesquisa Nacional de Demografia e Saúde da Criança e da Mulher - PNDS 2006: dimensões do processo reprodutivo e da saúde da criança. Brasília: MS; 2009.

10. Viellas EF, Domingues RMSM, Dias MAB, Gama SGN, Theme Filha MM, Costa JV, Bastos MH, Leal MC. Assistência pré-natal no Brasil. Cad Saude Publica 2014; 30(Supl.):S85-S100.

11. Beeckman K, Louckx F, Masuy-Stroobant, Downe S, Putman K. The development and application of a new tool to assess the adequacy of the content and timing of antenatal care. BMC Health Serv Res 2011; 11:213.

12. Ciceklioglu M, Soyer MT, Ocek AZ. Factors associated with the utilization and content of prenatal care in a western urban district of Turkey. Int J Qual Health Care 2005; 17(6):533-539.

13. Osungbade K, Oginni S, Olumide A. Content of antenatal care services in secondary health care facilities in Nigeria: implication for quality of maternal health care. Int J Qual Health Care 2008; 20(5):346-351.

14. Domingues RMSM, Hartz ZMA, Dias MAB, Leal MC. Avaliação da adequação da assistência pré-natal na rede SUS do Município do Rio de Janeiro, Brasil. Cad Saude Publica 2012; 28(3):425-437.
15. Carvalho VCP, Araújo TVB. Adequação da assistência pré-natal em gestantes atendidas em dois hospitais de referência para gravidez de alto risco do Sistema Único de Saúde, na cidade de Recife, Estado de Pernambuco. Rev Bras Saúde Matern Infant 2007; 7(3):309-317.

16. Almeida SDM, Barros MB. Equidade e atenção à saúde da gestante em Campinas (SP), Brasil. Rev Panam Salud Publica 2005; 7(1):15-25.

17. Coimbra LC, Figueredo FP, Silva AAM, Barbiere MA, Bettiol H, Caldas AJM, Mochel EG, Ribeiro VS. Inadequate utilization of prenatal care in two Brazilian birth cohorts. Braz J Med Biol Res 2007; 40(9):1195-1202.

18. Programa das Nações Unidas para o Desenvolvimento Brasil. Atlas do desenvolvimento humano no Brasil [acessado 2013 jul 15]. Disponível em: http://www. atlasbrasil.org.br/2013/perfil/sao-luis_ma.

19. Costa GRS, Chein MBC, Gama MEA, Coelho LSC, Costa ASV, Cunha CLF, Brito LMO. Caracterização da cobertura do pré-natal no Estado do Maranhão, Brasil. Rev Bras Enferm 2010; 63(6):1005-1009.

20. Instituto Brasileiro de Geografia e Estatística. Censo 2010. Rio de Janeiro; 2011. [acessado 2013 mar 8]. Disponível em: http://censo2010.ibge.gov.br.

21. Victora CG, Huttly SR, Fuchs SC, Olinto MT. The role of conceptual frameworks in epidemiological analysis: a hierarchical approach. Int J Epidemiol 1997; 26(1):224-227.

22. Lima S, Carvalho M, Vasconcelos AG. Proposta de modelo hierarquizado aplicado à investigação de fatores de risco de óbito infantil neonatal. Cad Saude Publica 2008; 24(8):1910-1916.

23. Associação Brasileira de Estudos Populacionais. Critério de classificação econômica Brasil. Belo Horizonte; 2011 [acessado 2013 ago 15]. Disponível em: www. abep.org/novo/FileGenerate.ashx?id=2.

24. Beeckman K, Louckx F, Putman K. Determinants of the number of antenatal visits in a metropolitan region. BMC Public Health 2010; 10:527.

25. Boerleider AW, Wiegers TA, Mannien J, Francke AL, Deville W. Factors affecting the use of prenatal care by non-western women in industrialized western countries: a systematic review. BMC Pregnancy Childbirth $2013 ; 13: 81$

26. Jong EIF, Jansen DEMC, Baarveld F, Schans CP, Schellevis FG, Reijneveld SA. Determinants of late and/or inadequate use of prenatal healthcare in high-income countries: a systematic review. Eur J Public Health 2012; 22(6):904-913.

27. Victora CG, Matijasevich A, Silveira MF, Santos IS, Barros AJD, Barros FC. Socio-economic and ethnic group inequities in antenatal care quality in the public and private sector in Brazil. Health Policy Plan 2010; 25(4):253-261.

28. Ribeiro ERO, Guimarães AMDN, Bettiol H, Lima DDF, Almeida MLD, Souza L, Silva AAM, Gurgel RQ. Risk factors for inadequate prenatal care use in the metropolitan area of Aracaju, Northeast Brazil. BMC Pregnancy and Childbirth 2009; 9(31):1-8.

29. Bailey BA. Partner violence during pregnancy: prevalence, effects, screening, and management. Int $J$ Womens Health 2010; 2:183-197. 
30. Nketiah-Amponsah EN, Senadza B, Arthur E. Determinants of utilization of antenatal care services in developing countries. Afr J Econ Manag Sci 2013; 4(1):58-73.

31. Zanchi M, Gonçalves CV, Cesar JA, Dumith SC. Concordância entre informações do Cartão da Gestante e do recordatório materno entre puérperas de uma cidade brasileira de médio porte. Cad Saude Publica 2013; 29(5):1019-1028.

Artigo apresentado em 31/10/2014

Aprovado em 30/07/2015

Versão final apresentada em 01/08/2015 